\title{
Le soutien de l'État aux coopératives de transport israéliennes, à l'origine de la perte des valeurs coopératives dans le contexte néolibéral
}

\section{Lost values and cooperatives: How state support doomed Israel's transportation cooperatives under a neo-liberal government}

\section{Yifat Solel}

Numéro 320, avril 2011

Le monde coopératif dans une économie plurielle : le problème de l'attractivité

Cooperatives in a plural Economy: The issue of their Attractiveness

URI : https://id.erudit.org/iderudit/1020907ar

DOI : https://doi.org/10.7202/1020907ar

Aller au sommaire du numéro

Éditeur(s)

Association Recma

ISSN

1626-1682 (imprimé)

2261-2599 (numérique)

Découvrir la revue

Citer cet article

Solel, Y. (2011). Le soutien de l'État aux coopératives de transport israéliennes, à l'origine de la perte des valeurs coopératives dans le contexte néolibéral. Revue internationale de l'économie sociale, (320), 43-51.

https://doi.org/10.7202/1020907ar

\section{Résumé de l'article}

Jusqu'en 2000, 97 \% des bus israéliens étaient gérés par des coopératives. Les coopératives de transport sont encore de loin les principaux opérateurs dans le domaine des transports publics du pays, mais l'orientation néolibérale du gouvernement a visé dès lors à créer un marché compétitif en encourageant leur démutualisation. Le secteur a été transformé en un marché fragmenté de monopoles de petite envergure, contrôlés par des sociétés capitalistes. En quoi les coopératives sont-elles concernées par la privatisation ? Les chantres de la privatisation en Israël défendent l'idée que ce ne sont pas seulement les entreprises d'Etat qui ne sont pas efficientes, mais plutôt l'ensemble des entreprises non capitalistes. La campagne publique initiée par le nouveau gouvernement suggérait que les employés du secteur public percevaient des salaires trop élevés pour un service de faible qualité et que les citoyens pourraient recevoir davantage en payant moins. 


\section{LE SOUTIEN DE L'ÉTAT AUX COOPÉRATIVES DE TRANSPORT ISRAÉLIENNES, À L'ORIGINE DE LA PERTE DES VALEURS COOPÉRATIVES DANS LE CONTEXTE NÉOLIBÉRAL}

* Docteur en droit, Van-Leer Institute, Jérusalem. Mél: ysollel @013.net.

* Cet article est dédié à mon grand-père, Zvi Solel, décédé I'an dernier. II fut le premier porte-parole de la coopérative de transport Dan.

\author{
par Yifat Solel*
}

Jusqu'en 2000, 97 \% des bus israéliens étaient gérés par des coopératives. Les coopératives de transport sont encore de loin les principaux opérateurs dans le domaine des transports publics du pays, mais l'orientation néolibérale du gouvernement a visé dès lors à créer un marché compétitif en encourageant leur démutualisation. Le secteur a été transformé en un marché fragmenté de monopoles de petite envergure, contrôlés par des sociétés capitalistes. En quoi les coopératives sont-elles concernées par la privatisation? Les chantres de la privatisation en Israël défendent l'idée que ce ne sont pas seulement les entreprises d'Etat qui ne sont pas efficientes, mais plutôt l'ensemble des entreprises non capitalistes. La campagne publique initiée par le nouveau gouvernement suggérait que les employés du secteur public percevaient des salaires trop élevés pour un service de faible qualité et que les citoyens pourraient recevoir davantage en payant moins**.
(1) Les lignes de chemin de fer sont peu nombreuses en Israël et, jusqu'aux années 90 , elles ne constituaient pas de réels moyens de transport de masse. usqu'en 2000, $97 \%$ des bus publics en Israël étaient gérés par des coopératives. Depuis les années 60, deux entreprises se partageaient le marché: Egged, avec $70 \%$ du marché et 4000 bus, et Dan, avec $27 \%$ et 1300 bus. Après la fondation de l'Etat d'Israël en 1948, les coopératives de transport sont devenues les principaux et quasiment les uniques exploitants de transports publics du pays. Elles le sont encore, et de loin ${ }^{(1)}$, mais plus pour longtemps, car les liens financiers étroits entre le gouvernement et les coopératives de transport, si bénéfiques pour les coopératives durant plus de cinquante ans, ont été remis en cause par les récentes politiques néolibérales.

Le gouvernement a en effet souhaité créer officiellement un marché compétitif, ce qui s'est traduit pratiquement par l'encouragement à la démutualisation des coopératives de transport afin de transformer le secteur des transports publics en un marché fragmenté de monopoles de petite envergure, contrôlés par des sociétés capitalistes. Dans les lignes de bus 
dites "privatisées ", le service n'est pas meilleur que celui offert par les coopératives de transport, mais les salaires sont plus bas, l'amplitude des horaires de travail est plus importante, le retour sur investissement plus profitable pour les actionnaires. Comment comprendre cette évolution? Après avoir rappelé rapidement la place de la coopération en général en Israël, la première partie détaille celle de la coopération de transport en particulier jusquaux années 90. La seconde partie montre comment l'imbrication étroite entre le gouvernement et les coopératives de transport, après avoir bénéficié à ces dernières durant des années, a précipité leur chute.

\section{Les coopératives de transport public en Israël}

(2) La question de savoir si en Israël les coopératives peuvent être décrites comme un " mouvement " pourrait être débattue. De mon point de vue, il y avait au moins cinq ou six mouvements coopératifs en Israël à cette époque: le mouvement des producteurs indépendants et des coopératives de consommateurs (Sefer Hamashbir Hamerkazi, 1958); les coopératives institutionnelles (usines, construction, logement et services sociaux), toutes membres du Hevrat H'ovdim; la société des travailleurs, dont les actionnaires étaient les membres de la convention des principaux syndicats Histradut (Daniel, 1972 et 1976; Kurland, 1947); les coopératives bancaires et de crédit (Ziv, 2010); et les coopératives agricoles. (3) Par exemple Hamashbir, une coopérative fondée pendant la Première Guerre mondiale afin d'assurer la fourniture des biens élémentaires à tous les Juifs résidents, et qui a continué par la suite à acheter des fournitures lorsque les prix étaient faibles pour les revendre sans réaliser de bénéfices lorsque les prix remontaient.
Sous mandat britannique (1920-1948), la plupart des services sociaux rendus au public par les institutions juives étaient fournis par les coopératives. Le nouvel Etat d'Israël les a intégrées comme si elles faisaient partie des organes nationaux officiels. On attendait des coopératives quelles s'engagent dans des missions nationales: les coopératives de consommateurs devaient ouvrir des magasins en périphérie, afin d'éviter la pénurie alimentaire ou vestimentaire; les usines coopératives pourvoyaient aux besoins de l'armée; les centres de coopératives pour les industries et les services devaient créer des usines coopératives pour les nouveaux immigrants, afin de résorber la crise du chômage à laquelle le pays se trouvait confronté.

\section{La place historique de la coopération en Israël}

Les coopératives de transport étaient une survivance d'une économie jadis florissante reposant sur le système coopératif. A la fondation d'Israël en 1948, 2200 coopératives enregistrées (pour une population de 650000 personnes) tenaient des positions dominantes dans tous les secteurs de l'économie. Au-delà des tensions inhérentes à toutes les coopératives entre leurs caractéristiques économiques et sociales, les coopératives de la Palestine pré-Israël se caractérisaient par un engagement patriotique fort (Sternell, 1995). Celui-ci s'est traduit tant dans la politique économique des coopératives que dans leur politique sociale. Chaque mouvement coopératif ${ }^{(2)}$ a intégré différemment ces éléments: pour certains, l'engagement national signifiait que les coopératives pouvaient mettre leurs gains financiers au service de la réalisation des objectifs nationaux ${ }^{(3)}$, alors que pour d'autres coopératives, le volet social de leurs activités a été sérieusement mis à mal.

De toutes les coopératives israéliennes, les coopératives de transport public étaient les plus orientées vers les affaires commerciales: elles privilégiaient en effet les objectifs économiques plutôt que des objectifs sociaux ambitieux. Le mouvement est né au cours des années 20, lorsque des groupes de chauffeurs, pour survivre, se sont structurés géographiquement en 
(4) Association générale des travailleurs de la terre d'Israël, fondée en 1920. Elle est à I'origine de l'unification des partis de la gauche sioniste qui a donné naissance au Mapaï, I'actuel Parti travailliste (NDLR). petites coopératives; grâce à un processus d'unification, celles-ci sont devenues plus importantes et plus puissantes. En 1948, il y avait cinq entreprises financièrement solides, qui prenaient soin de leurs membres et leur accordaient divers avantages en fonction des résultats de leur bilan annuel.

On distingue différents types de coopératives de transport, selon la nature de l'entité qui détient les actifs (tableau 1). Les coopératives israéliennes de transport appartiennent à la troisième catégorie. Les prestations mises en place pour les membres des coopératives incluaient leurs propres épiceries, des camps d'été pour les enfants, des activités sportives et culturelles et un certain nombre de quartiers d'habitation construits à l'usage des coopérateurs.

\section{Les relations avec le Histadrut}

Les relations entre les coopératives de transport et le Histadrut ${ }^{(4)}$ 'ont pas toujours été aussi cordiales que l'on pourrait le supposer. Contrairement aux autres coopératives, celles de transport ont refusé d'être soumises à l'ensemble des directives du Histadrut et ont menacé à plusieurs reprises de quitter à la fois ses institutions et son contrôle partiel. La plupart des conflits entre les coopératives de transport et le Histadrut étaient relatifs aux salaires et aux conditions sociales des travailleurs non membres des coopératives. En 1932, seulement $12 \%$ des travailleurs des coopératives n'étaient pas membres - tous les chauffeurs l'étaient -, mais en 1940 la proportion de travailleurs non membres atteignait les $30 \%$. Le Histadrut voulait limiter le travail des non-membres, mais les coopératives ont refusé, même si parmi les coopérateurs beaucoup estimaient que le travail salarié était contraire aux intérêts internes des coopératives. Le premier ministre des Transports, qui était l'ancien secrétaire général de l'organisation des travailleurs Histadrut, exigea des coopératives qu'elles mettent fin au travail salarié et qu'elles autorisent les travailleurs à les rejoindre. Le long débat qui s'ensuivit trouva sa conclusion dans un mécanisme

\section{Tableau 1}

Trois types de coopératives de transport

\begin{tabular}{|l|l|l|}
\hline Membres & Mission & Activité \\
\hline Consommateurs & $\begin{array}{l}\text { Satisfaction des besoins } \\
\text { de transport }\end{array}$ & $\begin{array}{l}\text { Emploi } \\
\text { de chauffeurs salariés }\end{array}$ \\
\hline Chauffeurs & Coopérative de services & $\begin{array}{l}\text { Exploitation de bus } \\
\text { appartenant à des tiers }\end{array}$ \\
\hline Chauffeurs & $\begin{array}{l}\text { Coopérative } \\
\text { de travailleurs }\end{array}$ & $\begin{array}{l}\text { Possession } \\
\text { et exploitation des bus }\end{array}$ \\
\hline
\end{tabular}


(5) En 1967, Hamekasher et Egged ont fusionné, ce qui a réduit à deux le nombre de coopératives de transport. bien organisé, qui permit aux travailleurs de longue date de devenir des coopérateurs et d'éviter que ne s'aggrave le ratio entre les membres et les salariés.

\section{Une concentration encouragée par l'Etat}

On l'a vu, il existait un lien naturel entre les coopératives et le premier gouvernement israélien. L'Etat avait besoin de mettre en place ses institutions, alors qu'il se trouvait en situation de conflit et que des dizaines de milliers de nouveaux immigrants rejoignaient un pays économiquement déprimé. Les rôles qui furent endossés par les coopératives, ou qui furent assignés aux coopératives par le gouvernement, étaient un mélange d'activités commerciales et de services publics, sans ligne définie qui aurait pu les distinguer les unes des autres. Ainsi, les coopératives de consommateurs avaient pris en charge la fourniture de biens et services dans les zones isolées et, à l'arrivée de l'hiver, elles s'engagèrent dans la distribution de vêtements et de chaussures dans les camps d'immigrants. Dans le cas des coopératives de transport, cela signifiait que le ministère des Transports était devenu un partenaire économique direct des coopératives. Les coopératives de transport, qui jusqu'ici n'avaient pas pu se permettre la moindre amélioration, eurent d'un coup les ressources pour acheter de nouveaux bus, construire des garages et augmenter les salaires de leurs membres.

Comme le gouvernement finançait les coopératives, il lui sembla légitime d'encourager leur fusion. Le besoin d'acquérir un grand nombre de véhicules contre des devises justifiait des achats en gros par le biais d'une seule coopérative. Cette solution permettait également de trouver plus facilement un équilibre entre les lignes de bus rentables et celles qui ne l'étaient pas, de fournir des infrastructures adaptées à l'essor de la fréquentation touristique et de la population nationale, notamment en trouvant des sources additionnelles de capitaux pour l'industrie des transports.

En 1952, il existait seulement trois coopératives de transport, l'une qui assurait le transport inter-cités: Egged, et les deux autres pour les villes principales: Hamekasher à Jerusalem et Dan à Tel-Aviv ${ }^{(5)}$.

En un mot, au cours des premières années d'Israël, la connexion entre le gouvernement et les coopératives de transport assura la prospérité des coopératives et, en même temps, elle renforça leur orientation sociale - un objectif que le gouvernement était prêt à financer. De ce fait, les coopératives acceptaient de nouveaux membres et leur offraient de meilleures conditions de travail. Leur stabilité financière, assise sur des fonds publics, conduisit les coopératives à ignorer les écarts grandissants entre leurs revenus commerciaux et leur budget coopératif - jusqu'au point où elles ne pouvaient plus envisager de survivre sans le soutien de l'Etat. Si l'imbrication étroite entre le gouvernement et les coopératives de transport a bénéficié à ces dernières durant des années, elle a aussi précipité leur chute. 


\section{La coopération de transport à l'épreuve du néolibéralisme}

Israël, qui avait été fondé sur un idéal autant socialiste que nationaliste, a pris lors des élections de 1977 un brusque virage à droite, qui s'est soldé par la mise en place d'une économie néolibérale. Le gouvernement de droite a transformé l'économie et éliminé la plupart des liens entre l'Etat et les coopératives, en même temps qu'il a réduit l'importance et la puissance des organisations de travailleurs. A la fin des années 70 et au début des années 80 , des dizaines de coopératives ont fait banqueroute, laissant des centaines de personnes au chômage, particulièrement dans les zones périphériques.

Les coopératives de transport ont miraculeusement survécu à la crise. Elles ont cependant été obligées de revoir les avantages sociaux de leurs membres, de réduire le nombre de congés payés, de diminuer les pensions versées et de limiter le droit à la retraite anticipée.

Les gouvernements travaillistes qui ont suivi n'ont entrepris aucun effort en vue de reconstruire un système orienté vers la justice sociale. Les coopératives de transport étaient quasiment les seules survivantes d'une économie qui reposait autrefois sur le modèle coopératif.

\section{L'époque de la privatisation}

Le 29 mai 1996, Netanyahu a été élu Premier ministre d'Israël. Ses croyances économiques se fondaient sur la supposition que le secteur public était trop riche et trop étoffé et que c'était le secteur privé qui portait à bout de bras tous ces " parasites ». Son autre obsession était que les syndicats étaient trop puissants et que les travailleurs gagnaient trop. Les accusations contre le secteur public et tous les autres secteurs réputés inefficaces ont fini par faire office de politique officielle. La conclusion immédiate était qu'il fallait tout privatiser.

Il serait pertinent de se demander ce que les coopératives ont à voir avec la privatisation, étant elles-mêmes des entreprises privées. Mais les chantres de la privatisation en Israël défendent l'idée selon laquelle ce ne sont pas seulement les entreprises d'Etat qui ne sont pas efficientes, mais plutôt l'ensemble des entreprises non capitalistes. La campagne publique initiée par le nouveau gouvernement suggérait que les employés du secteur public percevaient des salaires trop élevés pour un service de faible qualité et que les citoyens pourraient recevoir davantage en payant moins.

Cette fois, les coopératives de transport qui avaient survécu à la crise économique des années 80 ne furent pas laissées à l'écart. Seulement cinq mois après son arrivée au pouvoir, le $1^{\text {er }}$ janvier 1997 , le nouveau gouvernement décida d'ouvrir le secteur des transports publics à la concurrence en attribuant des autorisations à fournir des services de transport public dans des conditions concurrentielles. Selon le ministère des Finances (qui prend toutes les décisions économiques), les problèmes des transports publics découlaient du monopole de fait des coopératives, engendrant 
(6) La thèse selon laquelle un monopole est inefficace en toutes circonstances est un peu faible, mais il n'y a pas lieu de discuter ce point: le plan proposé ne contenait aucun élément concurrentiel. des coûts élevés en raison de la gestion par les membres selon des principes non lucratifs qui se traduisaient notamment par la compensation des lignes non rentables par les lignes rentables

\section{Une entrée en Bourse imposée}

Le ministère des Finances proposait d'extraire certaines lignes de bus du monopole coopératif pour transférer leur gestion à une compagnie privée qui en serait le seul exploitant, ce qui consistait à remplacer un monopole par un autre ${ }^{(6)}$. Le problème du financement croisé des lignes est également discutable. La rentabilité de la ligne est-elle le seul élément à prendre en compte lorsque l'on détermine le coût du ticket? Cette attaque était en réalité motivée par la volonté d'obtenir des informations sur les coûts des lignes, afin de déterminer lesquelles devraient être transférées aux sociétés capitalistes, en exigeant une comptabilité séparée des lignes. Les coûts élevés d'exploitation constituant un prétexte idéal pour changer les règles du transport public, le ministère a stigmatisé les salaires « disproportionnés » des membres de la coopérative (qui étaient en réalité plus faibles que les revenus moyens).

Derrière ces attaques, c'est l'idée même de gestion par les membres et la conception désintéressée de la coopérative qui étaient visées. Le ministre des Finances décida ainsi de contraindre la coopérative Egged à émettre des actions en Bourse en suspendant à cette condition le renouvellement du soutien gouvernemental acquis en vertu d'un accord vieux de cinquante ans.

\section{Des marchés publics interdits aux coopératives}

En février 1998, une offre fut rendue publique pour l'exploitation de vingt-deux lignes de bus, lesquelles étaient jusqu’à présent exploitées par les coopératives de transport, qui ne furent pas autorisées à faire une proposition, ni directement ni par l'intermédiaire de leurs filiales.

La coopérative Egged adressa une requête à la Cour suprême, car l'empêcher de postuler lui apparaissait anticonstitutionnel. La Cour suprême décida d'abroger l'offre, en raison de l'incapacité du gouvernement à mettre en place un plan concret et cohérent pour des réformes majeures dans le secteur du transport public. Toutefois, elle n'avait pas entendu la plainte des coopératives de transport arguant que l'Etat n'était pas autorisé à exclure leur candidature.

En mai 1999, l'administration des transports publia un plan directeur annonçant la mise en place de la concurrence dans le marché des transports publics. D'après le plan, 345 lignes de bus $(20 \%)$ devaient être retirées aux coopératives et soumises à des offres auxquelles les coopératives n'étaient pas autorisées à souscrire.

\section{Le gouvernement exige le licenciement des coopérateurs}

La dépendance des coopératives à l'égard des aides gouvernementales et les dettes colossales résultant du non-renouvellement des accords dans les 
(7) Rapport de la Cour des comptes 1-2002 sur les accords Egged-gouvernement relatifs à la concurrence dans le secteur des transports publics, publié en juin 2002. délais n'ont pas laissé d'autre choix aux coopératives que d'accéder aux exigences du gouvernement. Le soutien financier ne devait être octroyé à Egged qu'en échange d'une diminution du nombre de coopérateurs, soit par des mises à la retraite anticipées, soit en interdisant l'accès au sociétariat des travailleurs salariés. Pour bénéficier de cet appui, les coopératives devaient s'engager expressément à ne pas contester le prétendu processus concurrentiel dans le secteur des transports publics.

Afin d'atteindre le prétendu objectif de la concurrence, le gouvernement accepta de verser à Egged de 785 à 885 millions de shekels (160 à 180 millions d'euros). En outre, il consentit à verser une compensation de plusieurs centaines de milliers de shekels à chacun des membres des coopératives qui accepterait de partir en retraite anticipée.

Alors qu'au cours des années 80 et jusqu'au milieu des années 90 la part du financement de l'Etat dans les revenus des coopératives de transport était comprise entre 30 et $36 \%$, elle s'accrût de manière spectaculaire après les accords signés en 1996-1997 - la part des revenus octroyés par l'Etat atteignant $49 \%$-, notamment à cause du financement direct des accords de retraite anticipée des membres, ce qui força les coopératives à s'adapter.

Dans son rapport sur les accords signés entre le gouvernement et les coopératives de transport, le contrôleur d'Etat conclut qu'il n'existait aucune justification rationnelle ou économique à ces énormes dépenses publiques ${ }^{(7)}$.

\section{Les transports publics et le droit des travailleurs après la réforme}

Depuis l'année 2000, différentes sociétés ont répondu à des offres et obtenu des permis pour assurer des services de transport dans plusieurs zones d'Israël. Comme nous l'avons mentionné, il n'y a pas de concurrence, étant donné que chacune des compagnies de transport propose ses services dans un lieu déterminé en tant que seul fournisseur dans le district ou sur les lignes qui lui sont assignées. Les conditions des offres comprenaient en effet un volet concurrentiel seulement dans la phase de soumission. Les services requis étaient ainsi détaillés: le nombre de bus que l'offreur devrait posséder, les horaires qu'il devrait respecter, le type de tickets qu'il devrait vendre, etc. Les seuls aspects laissés à la négociation étaient les salaires et les avantages sociaux des travailleurs. Il y avait bien un critère relatif à la formation et aux titres des chauffeurs, mais celui-ci était sans incidence lors de l'examen des propositions, les seuls éléments considérés étant de nature financière.

Naturellement, ces instructions ont abouti à ce que toutes les compagnies choisies n'ont pas payé leurs travailleurs au-delà du salaire minimum et des prestations sociales légalement requises. Les chauffeurs enchaînaient de longues périodes de travail, n'avaient pas de sessions de formation et aucun débouché vers une augmentation de salaire ou une promotion en interne - les soumissions étant limitées à une période de six ans. Un groupe de travailleurs décida de joindre un syndicat et de négocier 
(8) Après l'augmentation, le salaire des chauffeurs est d'environ 1100 euros, alors que les chauffeurs de coopératives gagnent en moyenne 1600 euros. collectivement: la société lui répondit que, par principe, elle ne signerait pas d'accord collectif. Après des mois de grève au cours desquels le gouvernement soutint la société, les travailleurs furent contraints de se résoudre à des améliorations mineures, sans accord collectif.

Cela dit, il faut reconnaître que les offres publiées depuis 2009 comprennent une disposition relative aux salaires des travailleurs, ce qui, d'après le ministère des Transports, a entraîné une hausse de $25 \%$ du salaire des chauffeurs - mais celui-ci est encore largement inférieur au salaire moyen ${ }^{(8)}$. Les horaires longs, les conditions de travail difficiles, le manque de formation et l'absence de débouchés: rien de cela n'a changé.

\section{Conclusion: la fin de l'ère coopérative}

(9) En 2002, la coopérative Dan, en très mauvaise situation financière, a subi un processus de démutualisation pour devenir une société d'actionnaires. L'accord signé entre le gouvernement et la coopérative consistait en une offre financière généreuse et une garantie contre I'instabilité du fonds de pension des membres. Les membres s'étaient aussi vu promettre de recevoir des parts de propriété de la nouvelle société qui succéderait à la coopérative. Ainsi, après cinquante-sept années de services, la coopérative Dan avait cessé d'exister.

(10) On pourrait objecter que depuis des années la coopérative Egged n'était plus fondée sur les vrais principes coopératifs. Mais aussi dévoyées que soient les valeurs guidant une coopérative, celle-ci prend toujours en compte les droits des travailleurs, l'intérêt public, et elle est davantage orientée vers l'égalité que les entreprises capitalistes.
Egged, dernier survivant de l'époque des grandes coopératives en Israël (9), est au bord de la démutualisation ${ }^{(10)}$. La politique des gouvernements israéliens n'était pas en soi anticoopérative. Elle était opposée au droit des travailleurs et au pouvoir des travailleurs en tant que concept, un concept auquel la coopérative tient davantage que toute autre forme d'entreprise. De ce point de vue, la démutualisation des entreprises de transport est un grand succès - les chauffeurs des sociétés nouvellement fondées gagnent effectivement moins, ils n'ont pas de droits collectifs, pas d'opportunités de promotion et aucune sécurité de l'emploi. Les services auxquels le public accède ne sont pas meilleurs quauparavant, quand ils ne sont pas pires. C'est la raison pour laquelle seules les lignes hautement bénéficiaires ont été privatisées jusqu'à maintenant. Les implications à terme de ce processus, lorsque toutes les lignes de bus seront privatisées, ne sont pas encore connues, pas davantage que ne le sont l'impact d'une moindre formation des chauffeurs, des longs horaires de travail et des mauvaises conditions sur la sécurité routière et le taux d'accidents de véhicules.

La confiance des coopératives de transport dans la politique de l'Etat et le soutien de celui-ci leur ont donné pendant de nombreuses années une impression de stabilité financière. Cela leur a également permis de ne pas se préoccuper de leur solidité financière interne. Elles ont ainsi perdu leur indépendance financière. Aussi les coopératives se sont-elles retrouvées démunies lorsque les temps et les idéologies changèrent et que le gouvernement prit des décisions allant à l'encontre de leurs intérêts et de ceux des citoyens israéliens.

La fin de l'époque des coopératives symbolise la réalité actuelle de l'Etat d'Israël: un énorme fossé entre les riches et les pauvres, une classe moyenne en plein effondrement et une diminution des services publics. Il ne peut y avoir aucun doute: en ces temps de capitalisme néolibéral à outrance, personne ne peut avoir confiance en l'Etat. Lidée basique de construire une puissance économique alternative indépendante est pertinente aujourd'hui, comme elle l'était lorsque les coopératives israéliennes ont été crées, avant la fondation de l'Etat dont elles sont devenues dépendantes. 


\section{Bibliographie}

Daniel A., 1976, Labor enterprises in Israël, vol. 1-2, Jerusalem Academic Press.

Daniel A., 1972, Les coopératives, vision et réalisations, Am Oved publishing Ltd, Tel Aviv (en hébreu: Hacooperatzia, hazon vehagshama). Kurland S., 1947, Coopérative Palestine, the history of Histadrut, publiée par Sharon Books pour le Comité national Palestine pour le travail.

Sefer Hamashbir Hamerkazi 1955-1957,
1958, Le livre des sociétés coopératives de producteurs (en hébreu).

Sternell Z., 1995, Nation building or a new society? The Zionist Labor Movement (19041940) and the origins of Israel, Am Oved Publishing Ltd, Tel Aviv.

Ziv N., 2010, "Credit cooperatives in early Israëli Statehood: Financial institutions and social transformation ", Theoretical Inquiries in law, vol. 11-1. 